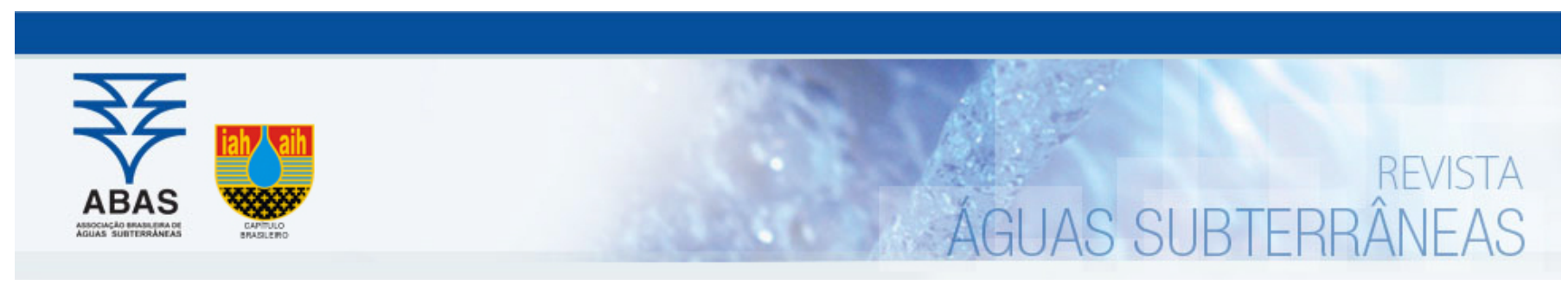

Estudos de Caso e Notas Técnicas

\title{
Avaliação de custos de sistema de remediação utilizando peroxone para tratamento de etenos clorados
}

\section{Evaluation of the costs of the remediation system using peroxone for the treatment of chlorinated ethenes}

\author{
Juan Felippe Santiago de Vasconcellos ${ }^{1}$; Fabiana Valéria da Fonseca1 ${ }^{凶}$ \\ 1 Universidade Federal do Rio de Janeiro, (UFRJ), Rio de Janeiro, RJ, Brasil. \\ 囚juan.vasconcellos@poli.ufrj.br; fabiana@eq.ufrj.br
}

Resumo

Palavras-chave:

Peroxone.

Ozônio.

Peróxido de hidrogênio.

Bombeamento e tratamento.

Etenos clorados.

\begin{abstract}
A utilização de Processos Oxidativos Avançados para remediação de áreas contaminadas tornou-se uma alternativa eficaz na destruição de contaminantes, seja na aplicação direta no solo e água subterrânea, ou através de sistemas de tratamento. Este trabalho teve como objetivo avaliar o custo de implantação e operação de um sistema de remediação Pump \& Treat utilizando peroxone como forma de tratamento para água subterrânea contaminada por etenos clorados. 0 sistema de remediação considerou as características hidrogeológicas da área de estudo e dos contaminantes. Foram considerados como custos de investimentos os relativos aos equipamentos e materiais necessários para a implantação do sistema de remediação, enquanto que os custos operacionais consideraram energia elétrica, peróxido de hidrogênio, mão de obra, manutenção, administração e análises laboratoriais da água subterrânea. Os resultados obtidos indicaram que cerca de $20 \%$ do custo de investimento está associado com a etapa de tratamento; o custo operacional foi de 551.484,86 (Ano 1), $R \$ 606.913,32$ (Ano 2) e R\$336.352,53 (Ano 3), demonstrando-se vantajoso em relação ao método tradicional de tratamento com carvão ativado.
\end{abstract}

Abstract

The use of Advanced Oxidation Processes for remediation of contaminated areas has become an effective alternative to the destruction of contaminants when applied directly to soil and groundwater or through the treatment systems. This paper aimed to evaluate the cost of investment and operating a Pump \& Treat remediation system to use peroxone as a treatment form for groundwater contaminated with chlorinated ethenes. The remediation system considered as hydrogeological characteristics of the study area and contaminants. The costs related to the equipment and materials necessary for the implementation of the system were considered as investment costs, while the operational costs considered electricity, hydrogen peroxide, labor, maintenance, administration, and laboratory analysis of groundwater. The results indicated that approximately $20 \%$ of the investment cost is associated with a treatment step and the operating cost step was $R \$ 551,484.86$ (Year 1 ), $R \$ 606,913.32$ (Year 2) and $\mathrm{R} \$ 336,352.53$ (Year 3), demonstrating an advantage over the traditional method of treatment with activated carbon.

DOI: http://dx.doi.org/10.14295/ras.v31i4.28951

\section{INTRODUÇÃO}

Uma das medidas de intervenção mais utilizadas no Brasil e no Mundo é o bombeamento e tratamento (Pump \& Treat), no qual consiste basicamente na extração da água subterrânea contaminada e no posterior tratamento, geralmente associado ao carvão ativado (CETESB, 2001).

Entre os diversos tipos de tratamento que existem, destacam-se os Processos Oxidativos Avançados (POA). Os POA aparecem como alternativa para o tratamento de efluentes e águas contaminadas por substâncias orgânicas de difícil degradação, tais como derivados do petróleo, compostos orgânicos voláteis e semi-voláteis, pesticidas e corantes têxteis (POYATOS et al., 2010; DENG \& ZHAO, 2015).

O processo baseia-se na geração de radicais livres, em geral radicais hidroxilas, que possuem alto poder de oxidação de compostos poluentes. Entre os POA, destacam-se o Fenton, a ozonização e a fotocatálise (radiação UV), com as técnicas podendo ser combinadas para aumento da eficiência na oxidação do poluente (POYATOS et al., 2010; DENG \& ZHAO, 2015).

Como os POA possuem a capacidade de mineralizar diversos poluentes orgânicos, ao longo dos últimos anos, passaram a ser utilizados na remediação de solo e água subterrânea contaminados 
por atividades industriais, comerciais ou agrícolas.

Contudo, o uso desses agentes oxidantes no meio subterrâneo deve ser feito de forma bem avaliada e controlada com o objetivo de evitar desperdícios de reagentes, a formação de compostos intermediários e acidentes de trabalhos causados pela manipulação equivocada do reagente (ITRC, 2005).

0 presente artigo tem como objetivo principal avaliar o custo de implantação e operação do Processo Oxidativo Avançado por peroxone $\left(\mathrm{H}_{2} \mathrm{O}_{2} / \mathrm{O}_{3}\right)$ no tratamento de água subterrânea contaminada por etenos clorados.

\section{2. Área de Estudo}

A área de estudo é uma antiga indústria química do setor farmacêutico, localizada no município do Rio de Janeiro, Região Metropolitana do Estado do Rio de Janeiro. A propriedade com 6.145 $\mathrm{m}^{2}$ de área possui histórico de ocupação por laboratórios e empresas farmacêuticas desde a década de 1970.
Entre 2014 e 2015 foram executadas investigações ambientais buscando atender a Resolução do Conselho Estadual de Meio do Rio de Janeiro $n^{\circ} 44$ (CONEMA, 2012). 0 diagnóstico ambiental realizado utilizou ferramentas tradicionais de investigação e identificou a contaminação da água subterrânea por tricloro eteno (TCE) e de seus subprodutos de degradação, 1,2 cis-dicloroeteno (1,2 cis-DCE) e cloreto de vinila (VC), os quais foram identificados em alguns poços com concentrações elevadas.

A pluma de TCE dissolvido em água subterrânea apresenta concentrações variando de 5 a $6.000 \mu \mathrm{g} / \mathrm{L}$, delimitada verticalmente em $9 \mathrm{~m}$ de profundidade, com comprimento aproximado de $58 \mathrm{x}$ $30 \mathrm{~m}$ e área de 1.193,254 m². Não foi observada a presença de NAPL no solo ou água subterrânea. A partir dos dados obtidos durante a investigação, o volume estimado de água contaminada foi de 2.613,23 $\mathrm{m}^{3}$ e o fator de retardamento, calculado com bases específicos da área de estudo causado pela sorção do local, foi de 5,9. A Figura 1 apresenta a Pluma de TCE em água subterrânea.

Figura 1 - Pluma de TCE em Água Subterrânea

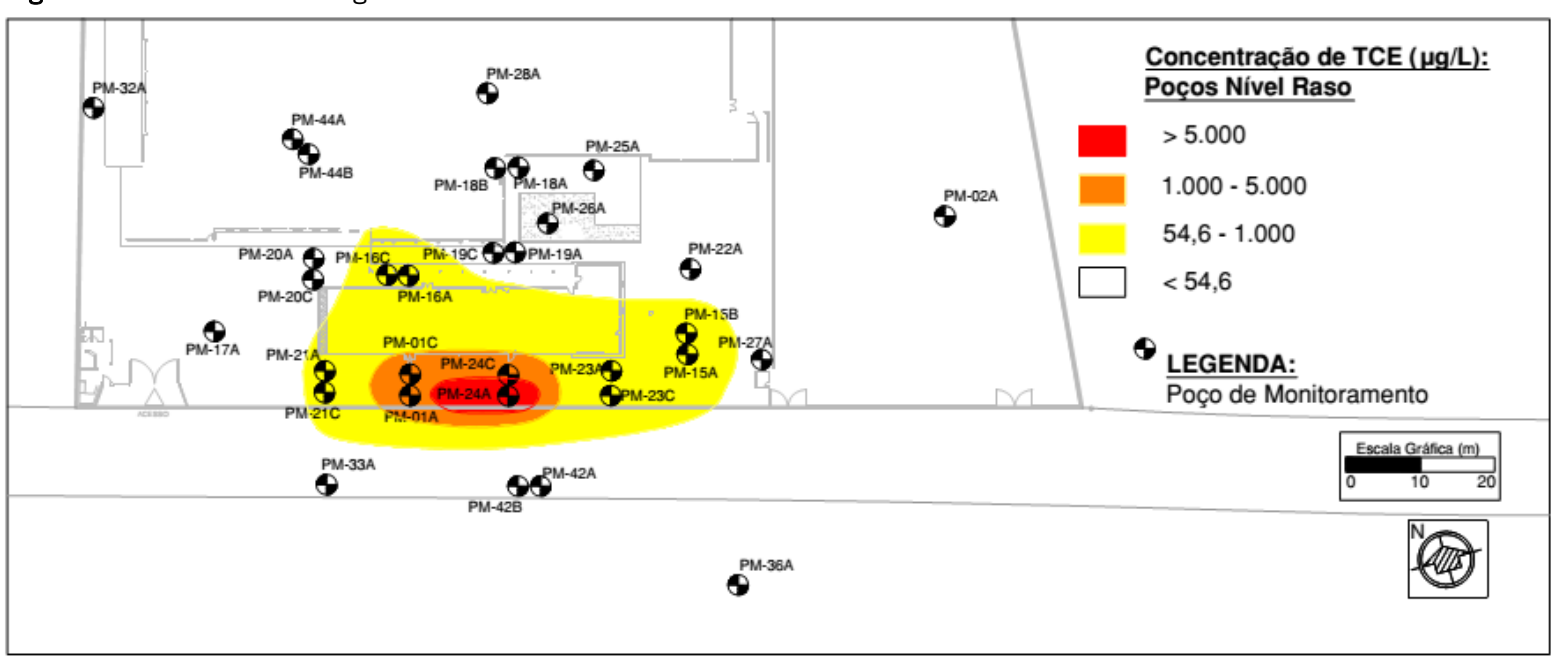

\section{MATERIAL E MÉTODO}

\subsection{Projeto de Remediação}

Foi selecionado o estudo realizado por YASUNAGA \& HIROTSUJ (2008) na degradação de TCE e 1,2 cis-DCE em água subterrânea por peróxido de hidrogênio e ozônio como referência para elaboração do projeto de remediação da área de estudo. Adicionalmente, o projeto considerou as características hidrogeológicas da área e os contaminantes presentes na água subterrânea (COHEN et al., 1997). O diagrama conceitual do sistema de remediação é apresentado na Figura 2.

O estudo realizado por YASUNAGA \& HIROTSUJ (2008) utilizou um dos métodos mais antigos de remediação, o Bombeamento e Tratamento (Pump \& Treat). Contudo, o tratamento foi realizado com os oxidantes peróxido de hidrogênio e ozônio, ao invés dos tradicionais filtros de carvão ativado (NAKANO et al., 2000).

O sistema de remediação projetado por YASUNAGA \& HIROTSUJ (2008) foi capaz de reduzir as concentrações iniciais de TCE e 1,2 cis-DCE de até $12 \mathrm{mg} / \mathrm{L}$ e $8 \mathrm{mg} / \mathrm{L}$, respectivamente, para con- centrações inferiores a 0,04 mg/L.

Os pontos de bombeamento de água subterrânea contaminada foram localizados estrategicamente na pluma de contaminação com os objetivos de remover a massa de contaminante e impedir a migração da pluma para as áreas residenciais vizinhas à área de estudo.

A água subterrânea tratada do sistema de remediação foi injetada no aquífero com objetivo de melhorar o controle hidráulico e auxiliar no tratamento da contaminação. O local previsto para a instalação dos poços de injeção foi a montante da pluma de contaminação.

Além disso, a utilização de ozônio no tratamento possibilita que as concentrações residuais de oxigênio dissolvido presentes no efluente possam auxiliar na mobilização dos contaminantes e na estimulação da biorremediação (COHEN et al., 1997).

Através de bombas pneumáticas instaladas dentro dos poços de bombeamento, a água subterrânea foi extraída com vazão média de $15 \mathrm{~L} / \mathrm{min}$ e passou por um filtro para remoção de partículas de solo. Posteriormente, foi adicionado o peróxido de hidrogênio 
no tanque reservatório para estimular a geração de radicais hidroxila durante a reação com o ozônio.

Após a adição de peróxido de hidrogênio, a água subterrânea foi transportada até um venturi para a injeção do ozônio e, em se- guida, passou por um misturador estático para melhorar o desempenho da transferência de massa do ozônio. A última etapa do tratamento foi o tanque de contato, por onde a água subterrânea permaneceu por cerca de 3 min antes de ser injetada no aquífero através dos poços de injeção.

Figura 2 - Diagrama do Sistema de Remediação

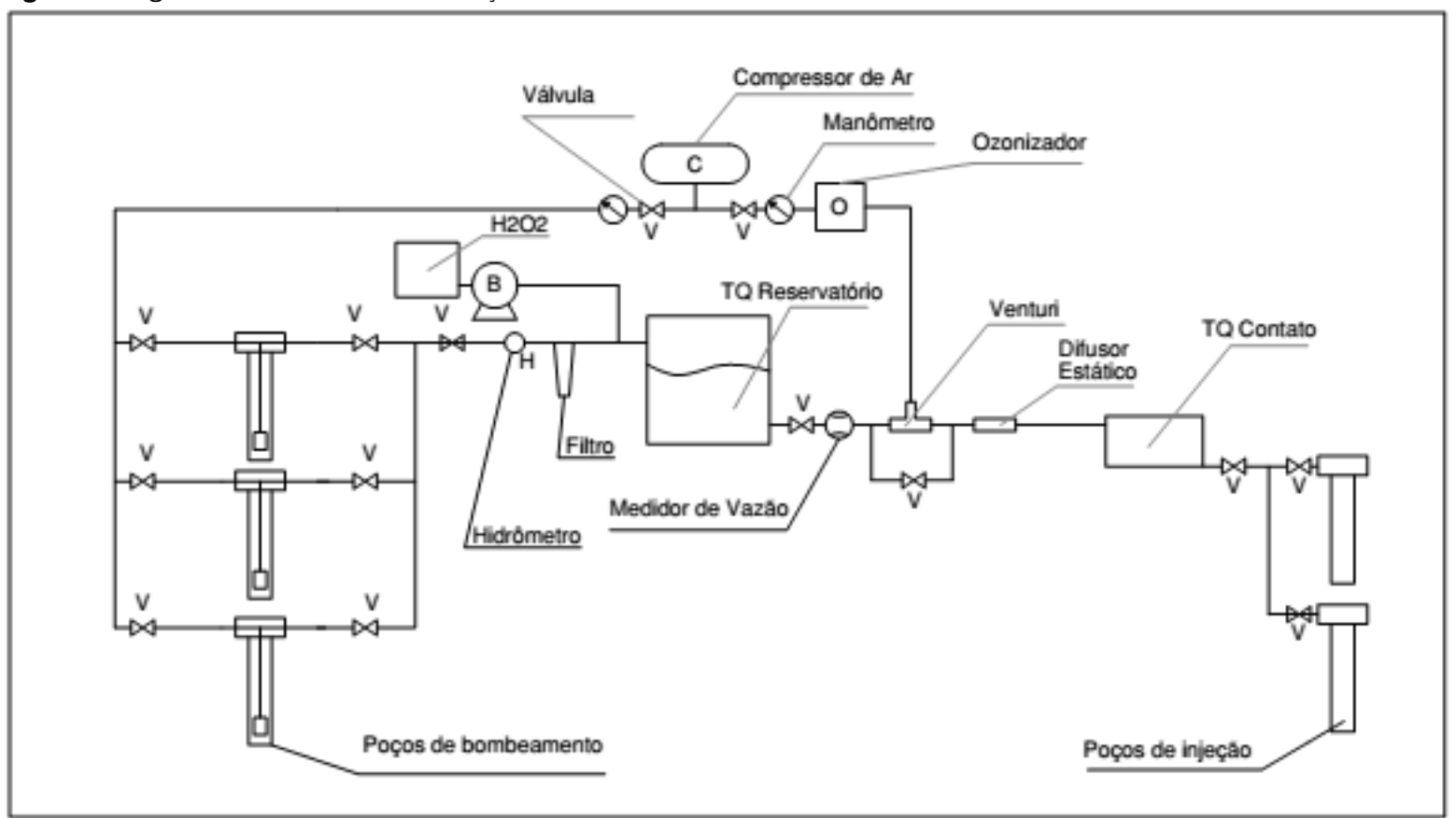

A Tabela 1 apresenta as condições de tratamento adotadas no projeto e no estudo de referência para a água subterrânea contaminada.
Com base nos resultados obtidos por YASUNAGA \& HIROTSUJ (2008) no experimento de campo, a dose inicial de ozônio planejada foi de $30,0 \mathrm{mg} / \mathrm{L}$ e a dose de peróxido de hidrogênio foi de $4,0 \mathrm{mg} / \mathrm{L}$, atendendo a razão ótima definida no estudo de 0,07-0,18 $\mathrm{mg} \mathrm{H}_{2} \mathrm{O}_{2} / \mathrm{O}_{3}$.

Tabela 1 - Condições de Tratamento da Água Subterrânea

\begin{tabular}{ccc}
\hline Parâmetros & Projeto & Yasunaga \& Hirotsuji (2008) \\
\hline Compostos Alvos & TCE; 1,2 cis-DCE; VC & TCE; 1,2 cis-DCE \\
Geração de ozônio (g/m3) & 80 & $70-80$ \\
Vazão de tratamento (L/min) & 15 & 2,5 \\
Concentração Inicial TCE (mg/L) & $4-6$ & $5-12$ \\
Concentração Inicial 1,2 cis-DCE (mg/L) & $1-10$ & $5-8$ \\
Concentração Inicial VC (mg/L) & $0-1$ & - \\
pH & $6-7$ & $6-7$
\end{tabular}

\subsection{Avaliação dos Custos}

As despesas envolvidas na remediação de áreas contaminadas são uma tarefa complexa durante a avaliação dos custos devido à dinâmica das variáveis que acarretam em alterações na operação dos sistemas de remediação.
De maneira geral, as despesas estão associadas aos custos iniciais de implantação, assim como os custos correntes e futuros, provenientes de operação e manutenção.

Os custos de investimento (CAPEX) foram divididos nos relativos ao bombeamento e injeção da água subterrânea e no tratamento 
da água subterrânea através da oxidação por peróxido de hidrogênio e ozônio. Dessa forma, é possível avaliar se o custo de implantação do tratamento de água subterrânea com ozônio e pe-

róxido de hidrogênio é relativamente alto comparado com o tratamento clássico com carvão ativado.

Foram considerados no levantamento do custo de operação e manutenção (OPEX), os custos com energia elétrica, produto químico (peróxido de hidrogênio), mão de obra, manutenção, administração e análises laboratoriais da água subterrânea.

De maneira geral, os custos de investimento foram obtidos com fornecedores especializados em equipamentos e materiais para utilização em áreas contaminadas, efluentes industriais e geração de ozônio.

\section{RESULTADOS E DISCUSSÃO}

O Sistema de Remediação foi composto por 6 bombas pneumáticas instalados em poços de bombeamento com $9 \mathrm{~m}$ de profundidade, onde foi considerada a vazão média de cada bomba em $2,5 \mathrm{~L} / \mathrm{min}$.

Em relação aos poços de injeção, foram instalados da mesma forma que os poços de bombeamento. Contudo, a injeção da água tratada foi por gravidade, com o objetivo de reduzir custos operacionais e reduzir impactos no fluxo da água subterrânea. Foi contemplada, no projeto, a instalação de quatro poços de injeção a montante da pluma com a mesma profundidade dos poços de bombeamento. A Figura 3 apresenta o layout do sistema de remediação planejado para a área.

Figura 3 - Layout do Sistema de Remediação

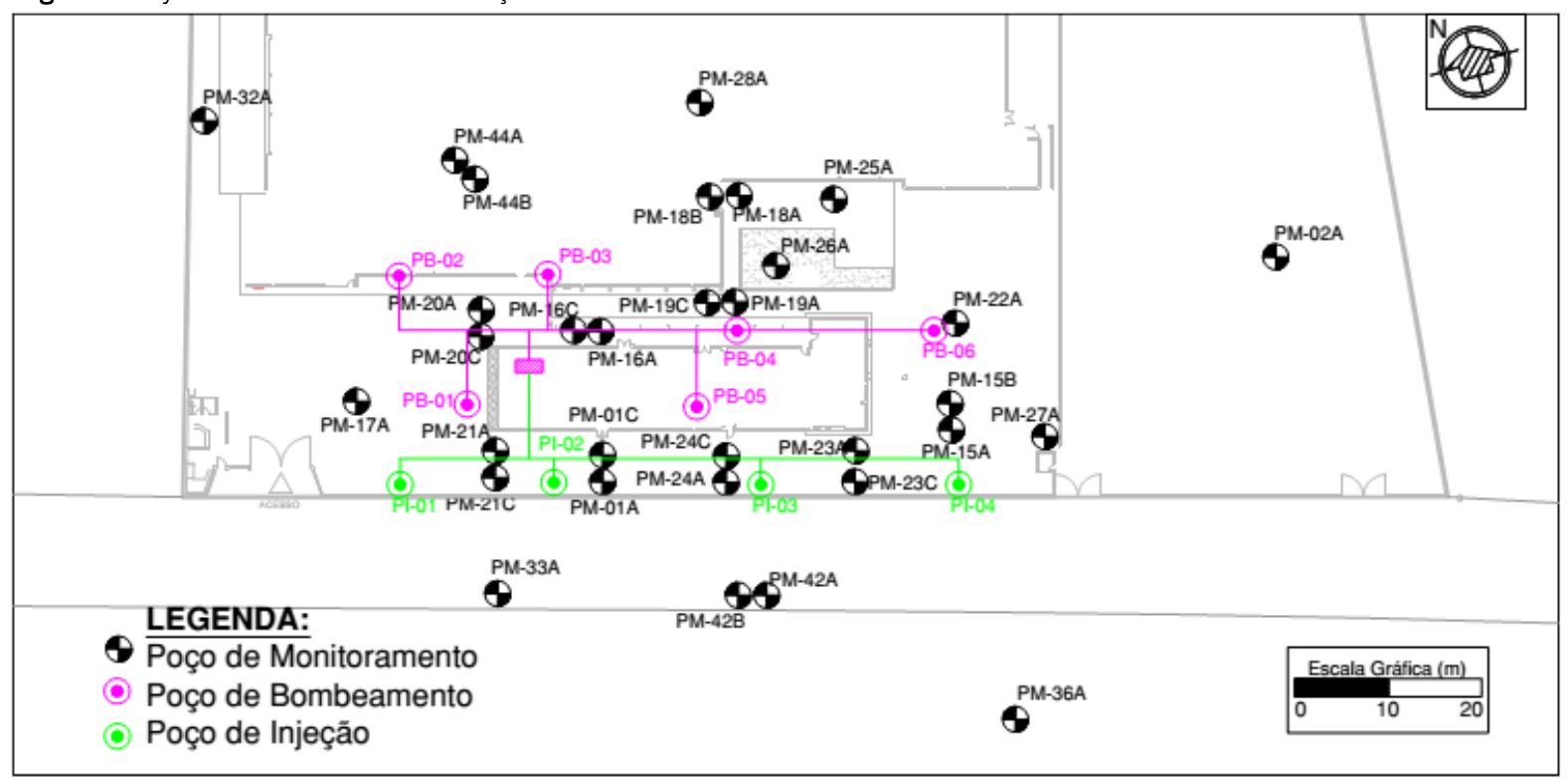

O tempo estimado para a remediação da área de estudo, considerando a possibilidade de sorção dos contaminantes no solo, foi obtido através da Equação 1 (DOMENICO \& SCHWARTZ, 1998).

$$
\mathbf{t}=\frac{\mathbf{R}_{\mathbf{F}} \mathbf{V}_{\mathbf{C}}}{\mathbf{Q}}
$$

Considerando que a vazão média de bombeamento do sistema foi de $15 \mathrm{~L} / \mathrm{min}$ e o fator de retardamento de 5,9, o tempo estimado necessário para bombeamento da fase dissolvida foi de aproximadamente 2 anos. Como fator de segurança, foram adicionados ao tempo estimado de remediação mais 6 meses de operação, totalizando, assim, 30 meses.
As Tabelas 2 e 3 apresentam o CAPEX dos materiais e equipamentos necessários para a instalação, assim como dos custos dos serviços de instalação dos poços de bombeamento e injeção, mão de obra especializada em montagem de sistema de remediação, assistência técnica na instalação do ozonizador e instalação elétrica dos equipamentos.

O custo total de investimento do Sistema de Remediação foi de $R \$ 254.619,31$, sendo aproximadamente $20 \%$, isto é, $R \$$ $51.256,94$ associado especificamente com os custos do tratamento com peróxido de hidrogênio e ozônio para vazão média de $15 \mathrm{~L} / \mathrm{min}$. 
Tabela 2 - Custo de Investimento com Equipamento e Material

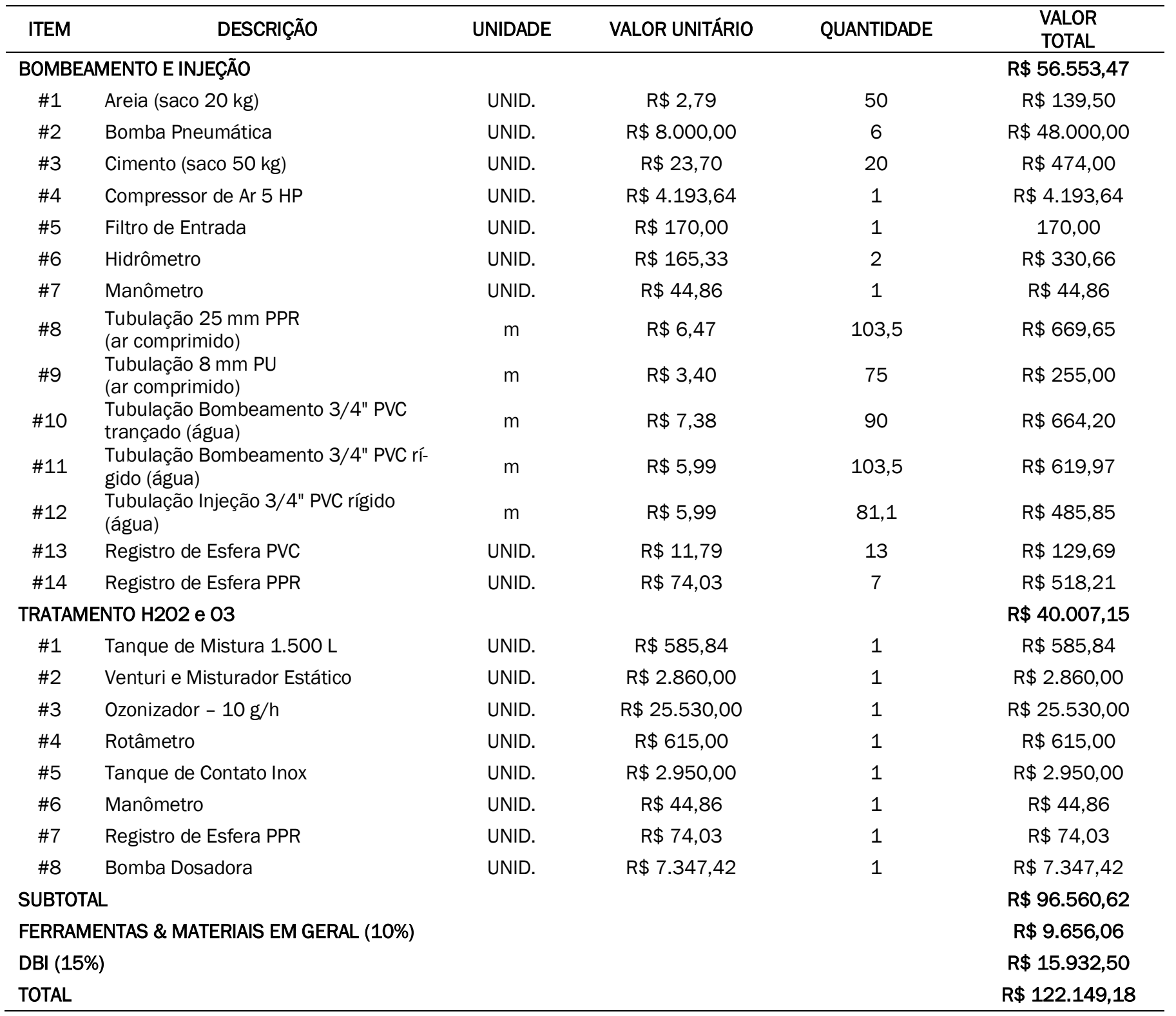

O custo é relativamente superior ao custo de investimento para tratamento de água subterrânea contaminada por etenos clorados com carvão ativado, onde geralmente são utilizados recipientes de aço inox com custo variando entre 5 a 20 mil reais, dependendo do modelo e capacidade de tratamento e carvão ativado granulado, custando na faixa de $R \$ 350,00$ sacos de $25 \mathrm{~kg}$.

O consumo mensal de energia elétrica do sistema de remediação estimado foi de 3.126,66 kWh, relativamente baixo, onde o compressor de ar é responsável pelo maior consumo $(2.774,00$
kWh), devido sua potência ser cerca de 8 vezes superior ao ozonizador (334,80 kWh).

A Tabela 4 apresenta os custos resumidos de operação e manutenção (OPEX) do Sistema de Remediação para o tempo de remediação calculado de 30 meses. 
Tabela 3 - Custo de Investimento com Serviço

\begin{tabular}{|c|c|c|c|c|}
\hline DESCRIÇÃO & UNIDADE & $\begin{array}{l}\text { VALOR UNITÁ- } \\
\text { RIO }\end{array}$ & QUANTIDADE & VALOR TOTAL \\
\hline \multicolumn{4}{|c|}{ INSTALAÇÃO POÇOS DE BOMBEAMENTO E INJEÇÃO } & $\mathrm{R} \$ 63.800,00$ \\
\hline Mobilização da Equipe & - & - & 1 & $\mathrm{R} \$ 7.400,00$ \\
\hline Perfuração mecanizada & $\mathrm{m}$ & $\mathrm{R} \$ 250,00$ & 90 & $\mathrm{R} \$ 22.500,00$ \\
\hline Instalação Poço & $\mathrm{m}$ & $\mathrm{R} \$ 260,00$ & 90 & $\mathrm{R} \$ 23.400,00$ \\
\hline Acabamento & UNID. & $\mathrm{R} \$ 150,00$ & 10 & $\mathrm{R} \$ 1.500,00$ \\
\hline Diária & UNID. & $\mathrm{R} \$ 600,00$ & 10 & $\mathrm{R} \$ 6.000,00$ \\
\hline Quebra de Piso & UNID. & $\mathrm{R} \$ 300,00$ & 10 & $\mathrm{R} \$ 3.000,00$ \\
\hline \multicolumn{4}{|l|}{ MONTAGEM DO SISTEMA } & $\mathrm{R} \$ 10.405,80$ \\
\hline Funcionário & mês & $\mathrm{R} \$ 3.500,00$ & 2 & $\mathrm{R} \$ 7.000,00$ \\
\hline Imposto e Encargos & \% mês & - & $73,43 \%$ & $\mathrm{R} \$ 5.140,10$ \\
\hline \multicolumn{4}{|l|}{ INSTALAÇÃO OZONIZADOR } & $\mathrm{R} \$ 960,00$ \\
\hline Assistência Técnica & hora & $\mathrm{R} \$ 120,00$ & 8 & $\mathrm{R} \$ 960,00$ \\
\hline \multicolumn{4}{|l|}{ INSTALAÇÃO ELÉTRICA } & $\mathrm{R} \$ 25.000,00$ \\
\hline Serviço & Projeto & - & 1 & $\mathrm{R} \$ 25.000,00$ \\
\hline \multicolumn{4}{|l|}{ SUBTOTAL } & $\mathrm{R} \$ 101.900,10$ \\
\hline \multicolumn{4}{|l|}{ DBI (30\%) } & $\mathrm{R} \$ 30.570,03$ \\
\hline \multicolumn{4}{|l|}{ TOTAL } & $\mathrm{R} \$ 132.470,13$ \\
\hline
\end{tabular}

Tabela 4 - OPEX do Sistema de Remediação 1

\begin{tabular}{lccc}
\hline \multicolumn{1}{c}{ DESCRIÇÃo } & CUSTO ANO 1 & CUSTO ANO 2 & CUSTO ANO 3 \\
\hline Energia elétrica & $\mathrm{R} \$ 31.839,78$ & $\mathrm{R} \$ 35.660,55$ & $\mathrm{R} \$ 19.969,91$ \\
Operador & $\mathrm{R} \$ 52.029,00$ & $\mathrm{R} \$ 56.191,32$ & $\mathrm{R} \$ 30.343,31$ \\
Manutenção & $\mathrm{R} \$ 12.223,48$ & $\mathrm{R} \$ 14.057,01$ & $\mathrm{R} \$ 8.434,20$ \\
Produto Químico (H202) & $\mathrm{R} \$ 255,04$ & $\mathrm{R} \$ 280,54$ & $\mathrm{R} \$ 154,30$ \\
Análise Química & $\mathrm{R} \$ 115.860,00$ & $\mathrm{R} \$ 127.446,00$ & $\mathrm{R} \$ 70.095,30$ \\
Destinação de Resíduos & $\mathrm{R} \$ 2.300,00$ & $\mathrm{R} \$ 2.530,00$ & $\mathrm{R} \$ 2.783,00$ \\
Relatório Técnico & $\mathrm{R} \$ 194.000,00$ & $\mathrm{R} \$ 213.400,00$ & $\mathrm{R} \$ 117.370,00$ \\
\multicolumn{1}{c}{ SUBTOTAL } & $\mathrm{R} \$ 408.507,30$ & $\mathrm{R} \$ 449.565,42$ & $\mathrm{R} \$ 249.150,03$ \\
& $\mathrm{R} \$ 142.977,56$ & $\mathrm{R} \$ 157.347,90$ & $\mathrm{R} \$ 87.202,51$ \\
\hline
\end{tabular}

Foi estimado um custo progressivo com manutenção, isto é, no primeiro ano de operação, $10 \%$ da despesa de investimento com equipamentos e materiais, no segundo $15 \%$ e no terceiro $20 \%$, devido o provável desgaste dos materiais requerendo substituição e assistência técnica especializada.

O volume mensal de peróxido de hidrogênio necessário para a operação do sistema de remediação é baixo, sendo inferior a 10 litros.

O custo mensal com análises químicas está associado à coleta da água subterrânea em três pontos do sistema de remediação para a avaliação mensal da operação do sistema e das concentrações dos parâmetros de interesse.
Com base em USEPA (1998), GOTTSCHALK et al. (2010) e CLAYTON et al. (2011), os parâmetros de interesse definidos foram: Demanda Bioquímica de Oxigênio (DBO), Demanda Química de Oxigênio (DQO), Carbono Orgânico Dissolvido (COD), Compostos Orgânicos Voláteis (VOC), metais dissolvidos prioritários da lista Resolução CONAMA (2009) nº 420 e bromato.

A análise dos parâmetros DBO, DQO, COD e VOC tem como objetivo avaliar a eficácia na oxidação dos compostos orgânicos presentes no efluente, estabelecer relação com a remoção dos etenos clorados e monitorar as concentrações de saída do sistema de remediação. 
A oxidação por peroxido de hidrogênio e ozônio pode impactar na mobilidade dos metais presentes naturalmente na água subterrânea (CLAYTON et al., 2011), sendo assim, a concentração dos metais dissolvidos prioritários da Resolução n 420 (CONAMA, 2009) e bromato no efluente foram avaliados mensalmente.

A periodicidade semestral de monitoramento dos poços foi definida com o objetivo de avaliar a variação sazonal das concentrações nos poços de interesse ao longo do ciclo hidrológico.

Foram contemplados no monitoramento analítico semestral 38 poços, sendo 32 poços de monitoramento e os 6 de bombeamento. Os parâmetros de interesse considerados no monitoramento semestral foram: DBO, DQO, COD, VOC, ânions (cloreto, nitrato e sulfato), gases leves (metano, etano e eteno), alcalinidade e bromato.

O número de diárias foi estabelecido considerando a estimativa de 4 poços amostrados por dia, além da adição de 2 dias de segurança para eventuais problemas que pudessem impedir a amostragem. Logo, foram consideradas aproximadamente $12 \mathrm{di}-$ árias.

Foi estimada apenas uma destinação por ano, devido ao baixo volume de resíduo gerado pela operação do sistema de remediação. E os custos dos relatórios técnicos foram estimados com base na complexidade da interpretação dos resultados.

0 custo operacional do sistema de remediação para o tempo estimado foi $\mathrm{R} \$ 551.484,86$ (Ano 1), $\mathrm{R} \$$ 606.913,32 (Ano 2) e R\$ $336.352,53$ (Ano 3), sendo o custo do ano 3 proporcional a 6 meses de operação. 0 reajuste do custo entre o ano 1 e 2 foi de aproximadamente $10 \%$.

Considerando a vazão de tratamento de $15 \mathrm{~L} / \mathrm{min}$, a capacidade anual de tratamento do Sistema de Remediação, desconsiderando as eventuais pausas no sistema, é de $7.884 .000 \mathrm{~L}$; o custo operacional de tratamento da água contaminada é de $R \$ 0,07 / \mathrm{L}$ (ano 1), R\$ 0,08/L (ano 2) e R\$ 0,09/L (ano 3).

O tratamento de água subterrânea contaminada por etenos clorados através de peroxone, em substituição ao tradicional tratamento com carvão ativado, demonstrou-se vantajoso. Embora requeira aumento do consumo de energia elétrica, esse aumento demonstrou-se baixo, cerca de $11 \%$ do total de energia consumido pelo sistema de remediação.

0 tratamento com peroxone, basicamente, não gera resíduos. A geração prevista de resíduos está associada com a manutenção do sistema e dos poços, o qual é inerente a qualquer sistema de remediação. Contudo, o tratamento com carvão ativado pode gerar o próprio carvão ativado como resíduo, aumentando os custos com destinação de resíduo e compra de carvão ativado.

Outro problema no uso do carvão ativado no tratamento de água subterrâneas contaminadas, segundo KILDUFF \& KARANFIL (2002) e MIYAKE et al. (2003), é a redução significativa na capacidade de adsorção do carvão ativado devido à presença natural de matéria orgânica em águas subterrâneas.

Embora a regeneração do carvão ativado seja uma opção cada vez mais utilizada, segundo MEZZARI (2002), as técnicas disponíveis ainda apresentam limitações e são economicamente inviáveis para pequenos sistemas.

\section{CONCLUSÃO}

O custo de investimento total para implantação do Sistema de Remediação foi de $\mathrm{R} \$ 254.619$,31, onde aproximadamente $20 \%$ $(R \$ 51.256,94)$ está relacionado à etapa de tratamento do sistema de remediação.

O custo operacional estimado foi de $\mathrm{R} \$ 551.484,86$ (Ano 1), $\mathrm{R} \$$ 606.913,32 (Ano 2) e R\$336.352,53 (Ano 3), indicando-se vantajoso em relação ao tradicional tratamento com carvão ativado devido os valores obtidos, destruição dos contaminantes alvos e baixa geração de resíduo.

\section{REFERÊNCIAS}

CLAYTON, W.S.; PETRI, B.G.; HULING, S.G. Fundamentals of ISCO Using Ozone. In: SIEGRIST, R.L.; CRIMI, M.; SIMPKIN, T.J. (eds.). Situ chemical oxidation for groundwater remediation. New York: Springer, Inc., 2011. Chapter 5 .

COHEN, R. M; MERCER, J. W.; GREENWALD, R. M.; BELJIN, M. S., 1997. Design guidelines for conventional pump and treat systems. Washington: Environmental Protection Agency, 1997.

COMPANHIA AMBIENTAL DO ESTADO DE SÃO PAULO (CETESB). Manual de gerenciamento de áreas contaminadas. São Paulo, Brasil, 2001.

CONSELHO ESTADUAL DE MEIO AMBIENTE (CONEMA). Resolução n. 44, de 14 de dezembro de 2012 - Dispõe sobre a obrigatoriedade da identificação de eventual contaminação ambiental do solo e das águas subterrâneas por agentes químicos, no processo de licenciamento ambiental estadual, 2012.

CONSELHO NACIONAL DO MEIO AMBIENTE (CONAMA). Resolução n. 420 , de 28 de dezembro de 2009. Dispõe sobre critérios e valores orientadores de qualidade do solo quanto à presença de substâncias químicas e estabelece diretrizes para o gerenciamento ambiental de áreas contaminadas por essas substâncias em decorrência de atividades antrópicas, 2009.

DENG, Y.; ZHAO, R., 2015. Advanced oxidation processes (AOPs). Wastewater Treatment. Current Pollution Reports, v. 1, p. 167 - 176, 2015.

DOMENICO, P.A.; SCHWARTZ, F.W. Physical and chemical hydrogeology. 2. Ed. New York: John Wiley and Sons, Inc., 1998.

GOTTSCHALK, C., LIBRA, J.A.; SAUPE, A.. Ozonation of water and waste water: a practical guide to understanding ozone and its applications. 2. ed. Weinheim, Alemanha: Wiley-VCH, 2010.

INTERSTATE TECHNOLOGY \& REGULATORY COUNCIL (ITRC). Technical and regulatory guidance for in situ chemical oxidation of contaminated soil and groundwater. 2. ed. Washington, United States, 2005.

KILDUFF, J. E.; KARANFIL, T. Trichloroethylene adsorption by activated carbon preloaded with humic substances: effects of solution chemistry. Water Research, v. 36, p. 1685-1698, 2002.

MEZZARI, A.M.Utilização de carvões adsorventes para o tratamento de efluentes contendo pesticidas. Dissertação (Mestrado)- Departamento de Engenharia Química e Alimentos. Universidade Federal de Santa Catarina. Florianópolis, 2002.

MIYAKE, Y; SAKODA, A.; YAMANASHI, H.; KANEDA, H.; SUZUKI, M. Activated carbon adsorption of trichloroethylene (TCE) vapor stripped from TCE-contaminated water. Water Research, v. 37, p. 1852-1858, 2003.

NAKANO, Y.; HUA, L. Q.; NISHIJIMA, W.; SHOTO, E.; OKADA, M. Biodegradation of trichloroethylene (TCE) adsorbed on granular activated carbon (GAC). Water Research, v, 34, n.17, p. 4139-4142, 2000.

POYATOS, J.M.; MUÑIO, M.M.; ALMECIJA, M.C.; TORRES, J.C.; HONTORIA, E.; OSORIO, F. Advanced oxidation processes for wastewater treatment: state of the art. Water, Air and Soil Pollution, v. 205, p. 187-204, 2010. 
USEPA. Technical protocol for evaluating natural attenuation of chlorinated solvents in ground water.cincinnati, EPA/600/R-98/128, 1998.

YASUNAGA, N; HIROTSUJI, J. Efficient decomposition of trichloroethylene (tce) in groundwater by ozone-hydrogen peroxide treatment. The Journal of the International Ozone Association, Ozone: Science and Engineering, v. 30 , p. $127-135,2008$. 